\title{
Integrated planning of grids and energy conversion units in municipal multi-energy carrier systems
}

\author{
Christopher Hauk ${ }^{*}$, Andreas Ulbig and Albert Moser \\ From The 10th DACH+ Conference on Energy Informatics \\ Virtual. 13-17 September 2021
}

\author{
*Correspondence: \\ c.hauk@iaew.rwth-aachen.de \\ Institute for High Voltage \\ Equipment and Grids, Digitalization \\ and Energy Economics, RWTH \\ Aachen University, Schinkelstrasse \\ 6, 52062 Aachen, Germany
}

\begin{abstract}
The ongoing energy transition requires the planning of low-emission municipal energy supply systems. These systems comprise distribution grids for electricity, gas, and heat, as well as energy conversion units such as heating systems. This paper presents a linear optimization model considering these elements in order to identify the cost-minimizing system design while achieving a given $\mathrm{CO}_{2}$ emission limit. The model is applied to an exemplary test case comprising 900 buildings. In order to increase scalability of the model to larger system sizes, the effect of reducing the spatial resolution on the optimization results is analyzed. The results show that the effect is small and that spatial aggregation is indeed a valid approach to reduce problem complexity and to allow significant speedups, reaching a factor of 200 for the given case study.
\end{abstract}

Keywords: Municipal energy supply, Multi-energy carrier systems, Sector coupling

\section{Introduction}

The ongoing energy transition requires the reduction of emissions attributed to the energy consumption of buildings, bringing municipal energy supply systems into focus. These systems include infrastructures for energy carriers such as electricity, gas, and heat, as well as conversion units such as heating technologies within the buildings. The holistic planning of municipal energy supply systems with numerous decision options requires mathematical optimization tools, which consider the expansion of infrastructures and conversion units in an integrated approach.

\section{Modeling of municipal energy supply systems}

The concept of energy hubs (Geidl 2007; Geidl et al. 2007) introduced the combination of multiple interconnected energy carriers into the optimization of energy systems. Since then, a wide variety of optimization models for municipal energy systems has been developed (Weinand et al. 2020; Keirstead et al. 2012; van Beuzekom et al. 2015; Allegrini et

(c) The Author(s). 2021 Open Access This article is licensed under a Creative Commons Attribution 4.0 International License, which permits use, sharing, adaptation, distribution and reproduction in any medium or format, as long as you give appropriate credit to the original author(s) and the source, provide a link to the Creative Commons licence, and indicate if changes were made. The images or other third party material in this article are included in the article's Creative Commons licence, unless indicated otherwise in a credit line to the material. If material is not included in the article's Creative Commons licence and your intended use is not permitted by statutory regulation or exceeds the permitted use, you will need to obtain permission directly from the copyright holder. To view a copy of this licence, visit http://creativecommons.org/licenses/by/4.0/. 
al. 2015; Mancarella 2014). These models can be differentiated based on their consideration of individual technologies or integrated systems, their focus on grid operation or grid expansion, their degree of abstraction in the modeling of grids, and the energy carriers considered.

A first group of models focuses on the integration of individual technologies such as thermal storages, CHP units, or heat pumps into a given cross-sectoral energy system (Bachmaier et al. 2015). The expansion of energy infrastructures is not considered. A second group of models focuses on the operational optimization of multi-energy carrier grids, again without consideration of their expansion planning (Geidl and Andersson 2007; Widl et al. 2015; Moeini-Aghtaie et al. 2014; Fichera et al. 2018; Martinez Cesena and Mancarella 2019). A third group of models considers the expansion of energy distribution capacities, but abstracts from realistic grid structures (Mainzer 2019; Eggers et al. 2015; Juroszek and Kudelko 2016; Ko et al. 2017; Jennings et al. 2014), so that for example the study area is divided into sub-areas, between which energy exchange capacities can be expanded. Different grid levels and the routing on those are neglected. In a fourth group with more detailed focus on the grid structure, some models plan the grid expansion for a subset of energy carriers like heat (Falke et al. 2016; Falke and Schnettler 2016), electricity and gas (Saldarriaga et al. 2013), or electricity and heat (Lu et al. 2017). Models such as DISTRICT-HP (Saad Hussein 2018) consider the expansion of distribution grids for electricity, gas, and heat, as well as the expansion of conversion units (Lu et al. 2017; Wouters et al. 2014; Mehleri et al. 2013; Wakui et al. 2014; Shabanpour-Haghighi and Seifi 2015). However, these models are only exemplarily applied to small study areas, which do not represent municipal scales and can therefore not answer questions for the overall system design.

\section{Contributions of this paper}

This paper follows two objectives. First, it introduces an optimization model for municipal energy supply systems, which considers the infrastructures for electricity, gas, and heat on different grid levels as well as the conversion units linking them. This model is applied to an exemplary test case including 900 buildings. Second, as the further scaling to even larger systems quickly leads to impractical computation times, this paper explores what effects the reduction in spatial resolution has on the overall system design results. In the following sections, the optimization model including its objective function and constraints is introduced and the spatial aggregation approach is described. Afterwards, the model is applied to a test case and the design results with and without spatial aggregation are compared.

\section{Optimization model}

\section{Model overview}

The target of the presented model is to identify the cost-optimized design of a municipal energy supply system. The model is formulated as a linear optimization problem (van Beeck 1999) minimizing the overall system costs, including costs for investment into grids and conversion units as well as costs for energy consumption during operation. It takes the perspective of a central planner, who has control of all investment and operational decisions within the municipal energy system. The optimization variables therefore comprise installed capacities for central and decentral conversion units, electric cables and 
transformers, gas pipes and pressure regulators, and heat pipes. Furthermore, the system operation, described by the input and output of conversion units as well as flows on grid elements, is optimized. This approach enables the identification of optimized system designs from a holistic perspective neglecting the barriers of split ownership and the influence of frequently changing energy regulations.

The investments and operation are subject to various constraints as further introduced in "Constraints" section. The model allows for a brown field approach by considering existing grid capacities in the optimization, or a green field approach by neglecting those.

The input comprises the geo-referenced grid structure, the supply task, weather data, as well as technical and economic parameters. The grid structure, defined as a graph with nodes and edges, describes possible infrastructure connections for each energy carrier. In case of a brown field optimization, existing capacities for each edge are defined. The grid structure also contains nodes for all buildings to be supplied. The supply task defines demand time series for electricity, space heat, and warm water to each building. The weather data contains time series for the global radiation and ambient air temperature. The technical and economic parameters include electric and thermal efficiencies and investment costs of energy conversion units as well as investment costs for grid elements such as cables, pipes, transformers, and pressure regulators. Furthermore, specific emission factors and price time series for each energy carrier are included. All aforementioned input time series are full-year data sets with a time resolution of 15 minutes per time step.

\section{Grid model}

Municipal energy supply systems contain grids for different energy carriers as well as conversion units such as heating technologies. These are either located in central locations to feed into the grid, or in buildings, where the demand occurs. The grid of each energy carrier comprises different grid levels. The electricity grid relies on the low voltage level to connect buildings with distribution stations, and the medium voltage level for linking those with substations (Sillaber 2016). Similarly, the gas system comprises a low pressure and the overlaid high pressure level (Cerbe 2016). Heating grids can be split into main and final distribution levels (Nussbaumer et al.). These two grid levels, in the following referred to as 'upper' and 'lower' level, are connected using transformers (electricity), pressure regulators (gas), and heat pipes (heat). Within the optimization model, the grid information for each energy carrier is described by a graph with nodes and edges, as visualized in Fig. 1. The nodes are classified as building nodes, central conversion nodes, grid nodes, connection nodes, and exchange nodes.

Building nodes are present in the lower grid level of each energy carrier and have a demand for electricity and heat, which must be fulfilled by the overall system. In a building node, conversion units can be installed to directly serve the demand, such as heat pumps, electric or gas heaters, pellet heaters, small cogeneration units, solar thermal units, and photovoltaic systems.

Central conversion nodes, linking the different energy carrier grids, are present in the upper grid level. They can convert energy from one carrier to another using similar technologies as used in building nodes, but in large scale, and with the difference that generated heat is fed into the heat grid. 


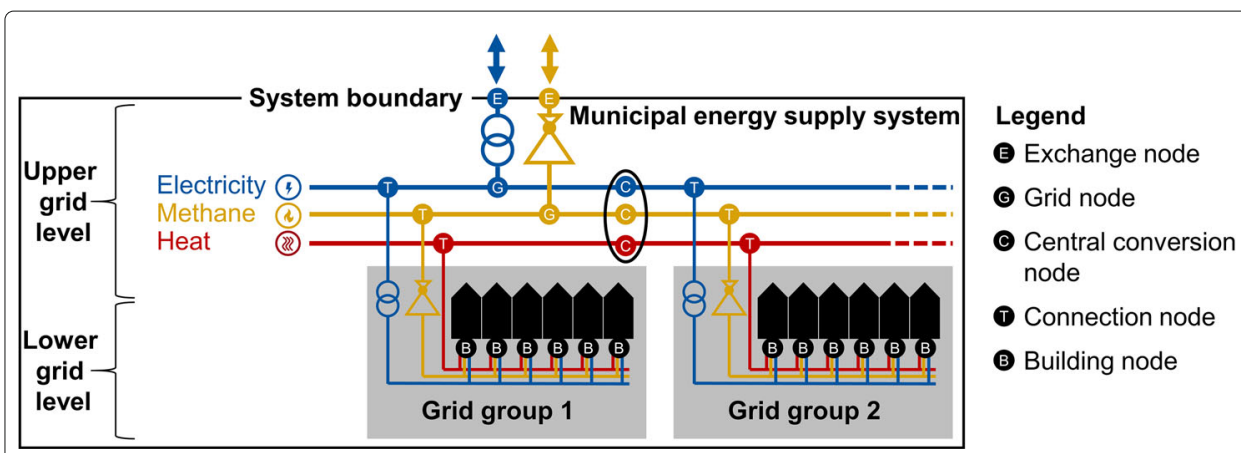

Fig. 1 The grid model contains individual nodes and edges for the energy carriers electricity, heat, and gas. 'Building nodes' and 'central conversion nodes' span across all energy carriers and connect these. The grid of each energy carrier consists of the 'upper' and 'lower' grid level connected at 'connection nodes'

Grid nodes and the edges between them exist on both grid levels and for each energy carrier individually. They perform the task of transporting their respective energy carrier between import nodes, central conversion nodes, and building nodes.

Connection nodes connect the upper and lower grid level of each energy carrier and represent electric transformers, gas pressure regulators, and heat connections.

The exchange nodes for the energy carriers electricity and gas are located in the upper grid level and allow the energy exchange between the modeled system and the outside. There is no exchange node for heat, as the heat grid is considered as being entirely local within this model.

The part of a grid on the lower level located under one connection node is referred to as 'grid group', as shown in Fig. 1. A building node belongs to the same grid group for all energy carrier grids.

\section{Objective function}

The objective function describes the minimization of annualized overall system costs with investment into grid and conversion units as well as operation, as stated by

$$
\begin{aligned}
Z & =C_{\text {grid,total }}^{\text {inv }}+C_{\text {conversion_units,total }}^{\prime \text { inv }}+C^{\text {operation }} \\
& =\sum_{c} \sum_{k} C_{c, k}^{\prime i n v}+\sum_{j} \sum_{n} C_{j, n}^{\prime i n v}+\sum_{c} \sum_{t} P_{c}^{\text {exchange }} p_{t, c}
\end{aligned}
$$

The first term $\sum_{c} \sum_{k} C_{c, k}^{\prime i n v}$ describes the annualized grid investment costs as sum across all energy carriers $c$ and all grid edges $k$ of the annualized investment costs per edge. These are derived based on the installed edge capacity, edge length, and annualized specific investment costs for the respective edge type. The second term $\sum_{j} \sum_{n} C_{j, n}^{\prime i n v}$ describes the annualized investment costs for conversion units as sum across all units $j$ in all nodes $n$ of the annualized investment costs per unit. These result from the installed unit capacity and annualized specific investment costs for the respective technology. The third term $\sum_{c} \sum_{t} P_{c, 0}^{\text {exchange }} p_{c, t}$ describes the operational costs for energy carrier consumption. These are defined as the sum across all time steps $t$ and energy carriers $c$ of the energy exchange at the exchange node multiplied by the price of the respective energy carrier. As the model takes the perspective of a central system planner, no charges related to energy regulations, 
such as network charges and energy taxes, are considered. The prices for the energy carriers therefore represent wholesale market prices, which in addition in the case of electricity inherently imply scarcity or abundance in the overall national system.

\section{Constraints}

\section{Technology constraints}

For each conversion unit $j$ in each node $n$ at each time step $t$, the energy carrier input and output are linked via the efficiency $\eta_{t, n, j}^{c}$ with

$$
P_{t, n, j}^{c, \text { out }}=P_{t, n, j}^{c, i n} \cdot \eta_{t, n, j}^{c} \quad \forall t, n, j
$$

The output of the unit $j$ in node $n$ is further limited by its installed capacity with

$$
P_{t, n, j}^{c, \text { out }} \leq \hat{P}_{n, j}^{c, \text { out }} \quad \forall t, n, j
$$

For heat pumps, the time-varying coefficient of performance (COP) replaces $\eta_{t, n, j}$. It is calculated based on time series for the flow temperature and evaporation temperature. For decentral air to water heat pumps, estimated building-specific flow temperatures and the outside air temperature are used in combination with the COP curve of a definable heat pump. For central water to water heat pumps, the flow temperature of the heating grid is regulated based on the outside temperature. The evaporation temperature, describing the temperature of the heat source such as ground water, waste water, or river water, is set at a constant value. A large-scale heat pump available on the market is used as basis for the COP curve.

\section{Node constraints}

Similar to the transport model described in Saad Hussein (2018), each grid node $n$ of each energy carrier $c$ must be in balance regarding incoming and outgoing edge flows $F_{t, m n}^{c}$, infeed $P_{t, n}^{c, \text { infeed }}$, and withdrawal $P_{t, n}^{c, \text { withdrawal }}$ in each time step $t$ :

$$
\sum_{(m, n)} F_{t, m n}^{c}-\sum_{(n, m)} F_{t, n m}^{c}+P_{t, n}^{c, \text { infeed }}-P_{t, n}^{c, \text { withdrawal }}=0 \quad \forall t, n, c
$$

In a building or central conversion node, multiple conversion, renewable energy, and storage units can be installed. Some units can feed their output into the grid, others can only supply within the same node. Therefore, a multi-layer node model is chosen, as shown in Fig. 2. Each building or central conversion node in each energy carrier grid is made up of three layers, whereby each layer has its own energy balance constraint.

Layer I represents the connection with the grid and is subject to Eq. 5. The balance equation of layer II considers the output from the subset $J_{n, g c}$ of conversion units in node $n$, which can feed their output back into the grid:

$$
\sum_{j \in J_{n, g c}} P_{t, n, j}^{c, \text { out }}+P_{t, n}^{c, \text { withdrawal }}-P_{t, n}^{c, \text { infeed }}-P_{t, n}^{c, \text { remain }}=0 \quad \forall t, n, c
$$

The term $P_{t, n}^{c \text {,remain }}$ describes the energy transferred from layer II to layer III. The balance equation of layer III considers the output from the subset $J_{n, n g c}$ of conversion units in node $n$, which cannot feed their output into the grid.

$$
\sum_{j \in J_{n, n g c}} P_{t, n, j}^{c, \text { out }}+P_{t, n}^{c, \text { remain }}-\sum_{j \in J_{n}} P_{t, n, j}^{c, i n}-P_{t, n}^{c, \text { demand }}=0 \quad \forall t, n, c
$$




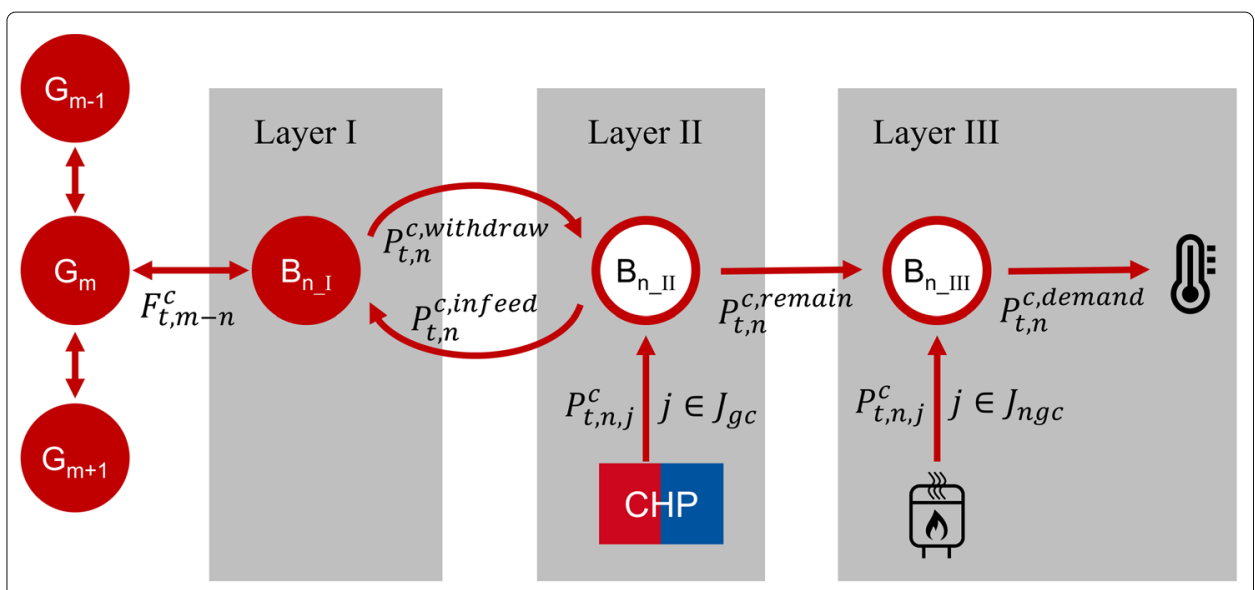

Fig. 2 Each building or central conversion node $n$ is modeled with three layers. Layer I represents the grid connection of node $n$, balancing withdrawal and infeed with the edge flows. Layer II considers the output from conversion units, which can feed their output into the grid, while layer III considers the output of all remaining units, the exogeneous energy demand and the input into all technologies in node $n$

This layer balances the energy demand of the building $P_{t, n}^{c, \text { demand }}$ and the fuel input into conversion units within the same node $P_{t, n, j}^{c, i n}$ on the one side with the energy transferred from layer II and the output of conversion units in layer III on the other side. The restriction of energy transfer between layer II and layer III to positive values hinders units installed in layer III from feeding their output into the grid.

$$
P_{t, n}^{c, \text { remain }} \geq 0 \quad \forall t, n, c
$$

\section{Grid constraints}

The flow $F_{t, m n}^{c}$ on each edge of energy carrier $c$ connecting nodes $m$ and $n$ and on each transformer (electricity) and pressure regulator (gas) is modeled as power flow neglecting parameters such as voltage, pressure, and temperature level, as well as losses. It is limited by its installed capacity:

$$
F_{t, m n}^{c} \leq \hat{F}_{m n}^{c} \quad \forall m, n, c, t
$$

\section{$\mathrm{CO}_{2}$ emission limit}

The overall system operation in all time steps is constrained by a $\mathrm{CO}_{2}$ emission limit, considering the emissions resulting from the consumption of energy carriers. For electricity and gas, the exchange across the system boundary at the exchange nodes is taken into account. A reverse electricity flow out of the system results in a $\mathrm{CO}_{2}$ credit. For pellets, the consumption at all nodes is considered. The specific emission factors for these energy carriers depend on the overall scenario parametrization.

\section{Aggregation of lower grid levels}

The "Grid model" section introduced the grid model of municipal energy supply systems with an upper and lower grid level. Results on the lower level allow for a detailed analysis of the optimal design for individual buildings and streets. Results for the upper level support assessments of the optimal overall cross-sectoral system design and are therefore in focus. The mere neglection of the lower level in the optimization, however, is not possible, as both levels interact with each other. At the same time, the full consideration of 
Table 1 Investment costs and thermal efficiencies for heat pumps (HP), electric heaters (EH), gas condensing heaters $(\mathrm{GH})$, pellet heaters $(\mathrm{PH})$, and combined heat and power units (CHP)

\begin{tabular}{llllllll}
\hline & EH & GH & HP_c & CHP_c & EH_c & GH_c & PH_c \\
\hline Inv. costs [EUR/kW] & 37 & 123 & 1200 & 359 & 17 & 58 & 210 \\
Thermal efficiency & 0.99 & 0.98 & $\mathrm{f}(\mathrm{t})$ & 0.43 & 0.99 & 0.98 & 0.94 \\
\hline
\end{tabular}

The '_c' represents central conversion technologies. The thermal efficiency of HP_c varies with time based on the COP curve of a commercial model (Emerson Climate Technologies 2012), the electric efficiency of CHP units is assumed as 43\%. The life span of all technologies is assumed to be 20 years

the lower level increases problem complexity significantly. The aggregation of information and thereby reduction of resolution on the lower level can help to bridge this conflict. The aggregation chosen in this work selects one representative building node for each grid group on the lower level and assigns the aggregated characteristics of all buildings within this grid group to it. For additive values such as the demand time series, the sum is calculated. For non-additive values such as the COP time series, the weighted average is calculated, with the heat demand time series used as weighting factors. The length of the new edge between the representative building and the connection node is calculated as the weighted average of all individual distances between the buildings and the connection node, with the annual energy demand as weighting factor.

\section{Exemplary application and results Description of the test case}

The introduced model is exemplarily applied to a test case based on a district in Aachen, Germany, including 900 multi-family houses, as visualized in Fig. 3a. The degrees of freedom include the installation of those conversion technologies introduced in Table 1, the grid expansion for electricity, heat, and gas, and the system operation. As the test case is located in a city center, heat pumps and pellet heaters in buildings are not provided as technology options. Furthermore, no storages are considered in this exemplary application. The technology parameters introduced in Table 1 have been defined based on a research of publicly available manufacturer data. Table 2 displays the parameters for grid expansion.

The synthetic grid data is created based on Open Street Map. The number of buildings, which can be supplied by one distribution station, is set to 50 based on standard transformer sizes and assumed electric power requirements of buildings. This results in 18 grid groups and connection nodes, which are spread accross the study area, and to which the buildings are assigned based on proximity. On the lower grid level, possible grid connections between building nodes and their respective connection node are defined based on a shortest path algorithm. These possible connections are assumed identical for all three energy carriers. On the upper grid level, possible electric connections in the form of

Table 2 Investment costs and life span for electric, gas, and heat edges in lower and upper grid level as well as transformers (electric) and pressure regulators (gas)

\begin{tabular}{lllllll}
\hline & el_lv & el_mv & gas_lp & gas_hp & heat_low & heat_up \\
\hline Inv. costs [EUR/(kW km)] & 630 & 23 & 79 & 4 & 242 & 60 \\
Life span [a] & 45 & 45 & 50 & 50 & 40 & 40 \\
& el_mv_lv & & gas_hp_lp & & & \\
Inv. costs [EUR/kW] & 40 & & 6 & & & \\
Life span [a] & 35 & & 45 & & \\
\hline
\end{tabular}


rings to link connection nodes with the exchange node are determined based on a traveling salesman algorithm. For gas, the same rings are defined and meshing edges added. The possible connections for heat on the upper grid level are determined based on the complete street network. Within the grid, three central conversion nodes are defined.

For the electric and hot water demand, probabilistic load profiles are generated for each building based on an estimated number of households, occupants' behavior, and the appliances in operation. Within this case study, the hot water demand is considered to be supplied by electric water heaters and is therefore added to the electric demand. For space heating, a thermal building model is applied, which translates the building's characteristics into an equivalent $\mathrm{RC}$ network.

The surrounding environment of the test case system is assumed as scenario 'TM80' in the German Energy Agency's 'dena Leitstudie' (Bründlinger et al. 2018) with a $\mathrm{CO}_{2}$ emission reduction target of $80 \%$ compared to 1990 . Accordingly, the specific emissions for electricity, gas, and pellets are set to $0.05,0.24$, and $0.01 \mathrm{~kg} \mathrm{CO}$-eq $/ \mathrm{kWh}$, respectively (Hecking et al. 2017). The emission reduction target of the test case system is assumed as comparably more ambitious and set to 95\% (base year 1990). The emissions in 1990 are calculated based on an assumed heating technology distribution dominated by gas and oil heaters (BDEW Bundesverband der Energie- und Wasserwirtschaft e.V. 2019) and the specific emissions for electricity, gas, and oil in 1990 with $0.76,0.24$, and $0.31 \mathrm{~kg} \mathrm{CO}_{2}$ eq/kWh (Bründlinger et al. 2018; Umweltbundesamt 2020). The $\mathrm{CO}_{2}$ limit results as $2.74 \mathrm{Mt} / \mathrm{a}$.

Using time series aggregation approaches (Teichgraeber and Brandt 2018; 2019), the annual input time series with a resolution of 15 min per time step are clustered to identify 120 representative time steps with a resolution of one hour per time step. These are considered in the optimization and their objective function coefficient is multiplied with a factor, which describes the number of original time steps represented.

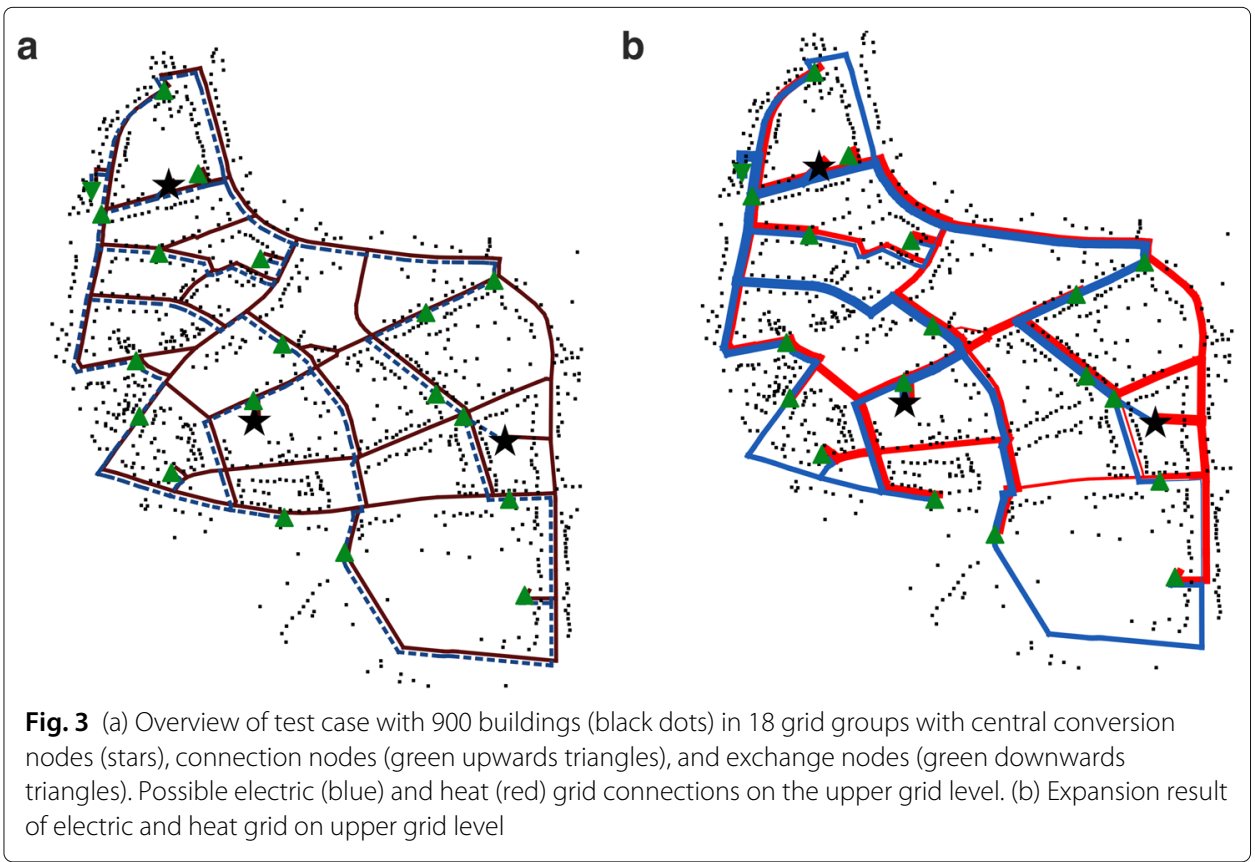


The optimization is performed as greenfield approach, once with high resolution with each building as individual node ('Disaggregated'), and once with low resolution with aggregated grid groups ('Aggregated'). The results are introduced in the following.

\section{Optimization with disaggregated grid}

The optimization with disaggregated grid results in an objective function value of 7.16 Million Euro and exhausts the emission limit fully. The resulting grid expansion on the upper grid level is visualized in Fig. 3b. The technology installation and operation for the entire system is visualized in the bar chart and duration curve in Fig. 4a. The base load is covered by central heat pumps and pellet heaters in combination with the heat grid, keeping $\mathrm{CO}_{2}$ emissions low. However, the installation of these technologies to cover peak loads would increase overall system costs significantly, so that central and decentral electric heaters are instead installed for this purpose. The share of central and decentral electric heaters is the result of a trade-off between additionally required heat and electric grid capacities as well as differing technology costs. No gas-fired technologies are installed, as the utilization of these would exceed the emission limit due to the specific emissions of methane in the above-introduced system scenario. The overall system design, combining different central and decentral technologies, demonstrates the benefits of hybrid heating systems.

The resulting heat grid starts from the central conversion nodes with capacities of up to $22 \mathrm{MW}$. The capacity decreases, as heat connection points to the lower grid groups are passed and supplied. The chosen network structure resembles a combination of meshes in the center and branches in the outer area. The electric grid capacities are expanded up to $12 \mathrm{MW}$ and are especially high between the exchange node and central conversion nodes with installed electric heaters. Since there are no gas-fired technologies installed, no gas grid is expanded.

\section{Optimization with aggregated grid and comparison of results}

The optimization with aggregated grid groups only requires $0.45 \%$ of computation time, a speedup by a factor of 200. It completes with an objective function value of 7.05
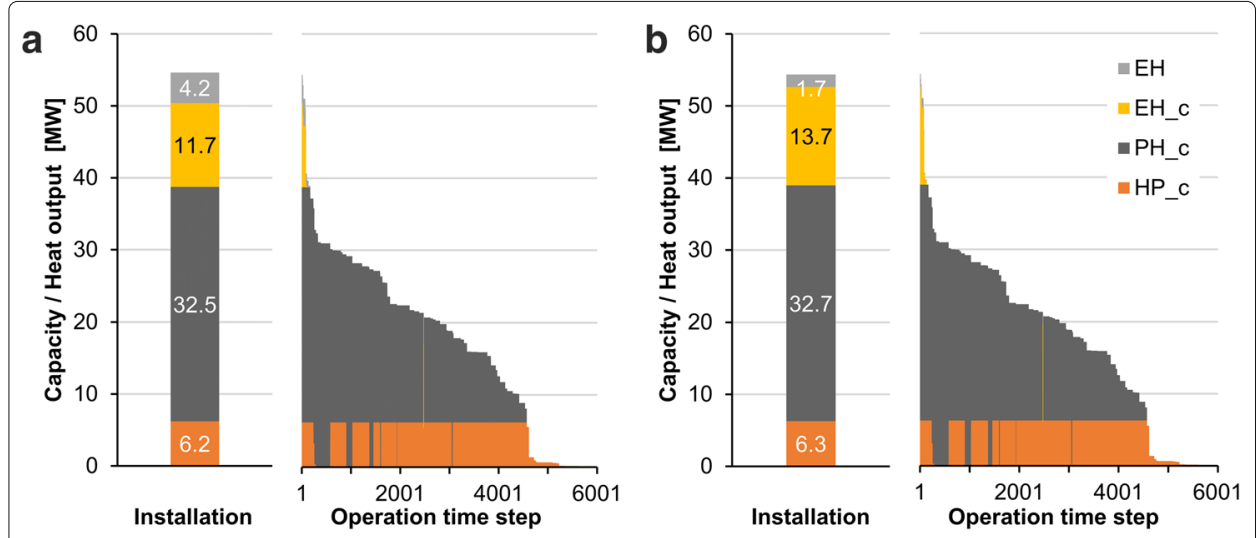

Fig. 4 Installed capacities and operation duration curves of heating units within the entire system. Central heat pumps (HP_c) and central pellet heaters (PH_c) provide base load, central and decentral electric heaters (EH_c and EH, respectively) provide peak load 
Million Euro, a deviation by $1.5 \%$ in comparison to the optimization with disaggregated grid, and also exploits the $\mathrm{CO}_{2}$ limit fully. The system design shows the utilization of the same heating technologies, as displayed for the installation and operation in Fig. 4b. While the selected heating technologies and their overall installed capacity is not affected by the aggregation to grid groups, the installed capacities for each technology differ. For central base load technologies, the aggregated optimization leads to $0.1 \mathrm{MW}$ and $0.2 \mathrm{MW}$ higher central heat pump and pellet heater capacities. It also results in $2 \mathrm{MW}$ higher central and 2.5 MW lower decentral electric heating capacity. Regarding the grid expansion, the same edges are used and similar capacities are installed across all energy carriers.

\section{Conclusion}

This paper presented a detailed model for the planning of municipal energy supply systems, including the distribution grids for electricity, gas, and heat, as well as the central and decentral energy conversion units. It is formulated as a linear optimization problem minimizing the overall system costs, while restricting the $\mathrm{CO}_{2}$ emissions to a predefined limit. To reduce problem complexity, an approach for spatial aggregation of buildings within the same grid group has been optionally applied. The application of this model to an exemplary test case comprising 900 buildings has shown the benefits of hybrid heating systems, utilizing multiple central and decentral conversion units. The comparison of optimization results with and without spatial aggregation has shown the same technology installations with small deviations in the installed capacities, as well as similar grid expansion results, while achieving a speedup by a factor of 200 . Therefore, the reduction of problem complexity with spatial aggregation is a possible approach, if the optimization purpose is the identification of design characteristics on the overall system level. Future work will introduce binary decision variables for grid expansion to the model and will present its application to larger system scales.

\section{About this supplement \\ This article has been published as part of Energy Informatics Volume 4 Supplement 3, 2021: Proceedings of the 10th DACH+ Conference on Energy Informatics. The full contents of the supplement are available online at https://energyinformatics. springeropen.com/articles/supplements/volume-4-supplement-3.

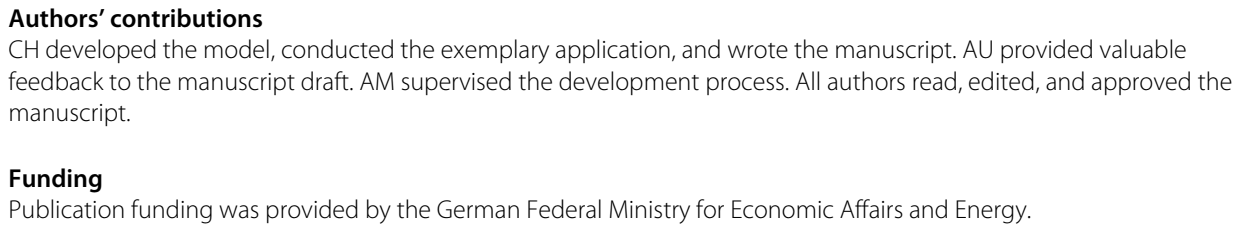

\section{Declarations}

Competing interests

The authors declare that they have no competing interests.

Published: 13 September 2021

\section{References}

Allegrini J, Orehounig K, Mavromatidis G, Ruesch F, Dorer V, Evins R (2015) A review of modelling approaches and tools for the simulation of district-scale energy systems. Renew Sust Energ Rev 52:1391-1404

Bachmaier A, Narmsara S, Eggers J-B, Herkel S (2015) Spatial distribution of thermal energy storage systems in urban areas connected to district heating for grid balancing. Energy Procedia 73:3-11 
BDEW Bundesverband der Energie- und Wasserwirtschaft e.V. (2019) Entwicklung des Wärmeverbrauchs in Deutschland. https://www.bdew.de/media/documents/Awh_20190529_Waermeverbrauchsanalyse_jwZ6BGZ.pdf. Accessed 03 May 2021

Bründlinger T, König JE, Frank O, Gründig D, Jugel C, Kraft P, Krieger O, Mischinger S, Prein P, Seidl H, Siegemund S, Stolte C, Teichmann M, Willke J, Wolke M (2018) dena-Leitstudie Integrierte Energiewende. Grey Literature, Berlin

Cerbe G (2016) Grundlagen der Gastechnik: Gasbeschaffung - Gasverteilung - Gasverwendung. 8vollständig überarbeitete auflage. Hanser Carl, München. http://www.hanser-fachbuch.de/9783446449657

Eggers J-B, Stryi-Hipp G, Herkel S (2015) A spatial resolution in four levels for a techno-economic municipal energy system model. In: Mathur J, Garg V (eds). Building Simulation 2015. BS Publications, Hyderabad. pp 2486-2493

Emerson Climate Technologies (2012) The High Temperature Ammonia Heat Pump. https://ammonia21.com/files/ 448_dvi143_neatpump_en_1209.pdf. Accessed 03 May 2021

Falke T, Krengel S, Meinerzhagen A-K, Schnettler A (2016) Multi-objective optimization and simulation model for the design of distributed energy systems. Appl Energy 184:1508-1516

Falke T, Schnettler A (2016) Investment planning of residential energy supply systems using dual dynamic programming. Sustain Cities Soc 23:16-22

Fichera A, Pluchino A, Volpe R (2018) A multi-layer agent-based model for the analysis of energy distribution networks in urban areas. Phys A: Stat Mech Appl 508:710-725

Geidl M (2007) Integrated modeling and optimization of multi-carrier energy systems. PhD thesis, ETH Zurich. https://doi. org/10.3929/ethz-a-005377890

Geidl M, Andersson G (2007) Optimal power flow of multiple energy carriers. IEEE Trans Power Syst 22(1):145-155

Geidl M, Koeppel G, Favre-Perrod P, Klockl B, Andersson G, Frohlich K (2007) Energy hubs for the future. IEEE Power Energy Mag 5(1):24-30

Hecking H, Hennes O, Elberg C, Frings C, Hintermayer M, Lencz D, Wildgrube T, Oschatz B, Winiewska B, Mailach B, Holm A, Kagerer F (2017) Szenarien für eine marktwirtschaftliche Klima- und Ressourcenschutzpolitik $2050 \mathrm{im}$ Gebäudesektor. Grey Literature, Berlin

Jennings M, Fisk D, Shah N (2014) Modelling and optimization of retrofitting residential energy systems at the urban scale. Energy 64:220-233

Juroszek Z, Kudelko M (2016) A model of optimization for local energy infrastructure development. Energy 96:625-643

Keirstead J, Jennings M, Sivakumar A (2012) A review of urban energy system models: Approaches, challenges and opportunities. Renew Sust Energ Rev 16(6):3847-3866

Ko W, Park J-K, Kim M-K, Heo J-H (2017) A multi-energy system expansion planning method using a linearized load-energy curve: A case study in South Korea. Energies 10(10):1663

Lu J, Bernreuther M, Volkwein S (2017) Mathematical Modeling and Global Optimization of Hybrid Energy Supply Networks, Vol. 358. Bibliothek der Universität Konstanz, Konstanz

Mainzer K (2019) Analyse und Optimierung Urbaner Energiesysteme: Entwicklung und Anwendung Eines Übertragbaren Modellierungswerkzeugs zur Nachhaltigen Systemgestaltung, PhD Thesis. Karlsruhe Institute of Technology. https:// doi.org/10.5445//R/1000092481

Mancarella P (2014) MES (multi-energy systems): An overview of concepts and evaluation models. Energy 65:1-17

Martinez Cesena EA, Mancarella P (2019) Energy systems integration in smart districts: Robust optimisation of multi-energy flows in integrated electricity, heat and gas networks. IEEE Trans Smart Grid 10(1):1122-1131

Mehleri ED, Sarimveis H, Markatos NC, Papageorgiou LG (2013) Optimal design and operation of distributed energy systems: Application to greek residential sector. Renew Energy 51:331-342

Moeini-Aghtaie M, Abbaspour A, Fotuhi-Firuzabad M, Hajipour E (2014) A decomposed solution to multiple-energy carriers optimal power flow. IEEE Trans Power Syst 29(2):707-716

Nussbaumer T, Thalmann S, Jenni A, Ködel J Planungshandbuch Fernwärme. http://www.verenum.ch/Dokumente/PLHFW_V1.2.pdf/ Accessed 30 April 2021

Saad Hussein N (2018) A method for evaluating building retrofit effects on a decentral energy system by a sector coupling operation and expansion model. Energy Syst 9(3):605-645

Saldarriaga CA, Hincapie RA, Salazar H (2013) A holistic approach for planning natural gas and electricity distribution networks. IEEE Trans Power Syst 28(4):4052-4063

Shabanpour-Haghighi A, Seifi AR (2015) Multi-objective operation management of a multi-carrier energy system. Energy 88:430-442

Sillaber A (2016) Leitfaden zur Verteilnetzplanung und Systemgestaltung: Entwicklung Dezentraler Elektrizitätssysteme. Springer Vieweg, Wiesbaden. http://www.springer.com/

Teichgraeber H, Brandt AR (2018) Systematic comparison of aggregation methods for input data time series aggregation of energy systems optimization problems. In: 13th International Symposium on Process Systems Engineering (PSE 2018). Elsevier, Amsterdam Vol. 44. pp 955-960

Teichgraeber H, Brandt AR (2019) Clustering methods to find representative periods for the optimization of energy systems: An initial framework and comparison. Appl Energy 239:1283-1293

Umweltbundesamt (2020) Strom- und Wärmeversorgung in Zahlen. https://www.umweltbundesamt.de/themen/klimaenergie/energieversorgung/strom-waermeversorgung-in-zahlen?sprungmarke=Strommix\#Strommix. Accessed 30 Apr 2021

van Beeck NMJP (1999) Classification of energy models. FEW Research Memorandum 777:1-25. Operations research

van Beuzekom I, Gibescu M, Slootweg JG (2015) A review of multi-energy system planning and optimization tools for sustainable urban development. In: 2015 IEEE Eindhoven PowerTech. IEEE, New York City. pp 1-7

Wakui T, Kinoshita T, Yokoyama R (2014) A mixed-integer linear programming approach for cogeneration-based residential energy supply networks with power and heat interchanges. Energy 68:29-46

Weinand JM, Scheller F, McKenna R (2020) Reviewing energy system modelling of decentralized energy autonomy. Energy 203:117817

Widl E, Muller W, Basciotti D, Henein S, Hauer S, Eder K (2015) Simulation of multi-domain energy systems based on the functional mock-up interface specification. In: 2015 International Symposium on Smart Electric Distribution Systems and Technologies (EDST). IEEE, New York City. pp 510-515 
Wouters C, Fraga ES, James AM, Polykarpou EM (2014) Mixed-integer optimisation based approach for design and operation of distributed energy systems. In: 2014 Australasian Universities Power Engineering Conference (AUPEC). IEEE, New York City. pp 1-6

\section{Publisher's Note}

Springer Nature remains neutral with regard to jurisdictional claims in published maps and institutional affiliations.

Submit your manuscript to a SpringerOpen ${ }^{\circ}$ journal and benefit from:

- Convenient online submission

Rigorous peer review

- Open access: articles freely available online

- High visibility within the field

- Retaining the copyright to your article

Submit your next manuscript at $\gg$ springeropen.com 